\title{
Evaluating the Effectiveness of an Improved Active Dynamic Signage System using Full Scale Evacuation Trials
}

\author{
EDWIN R GALEA, HUI XIE, STEVEN DEERE, DAVID COONEY and LAZAROS FILIPPIDIS \\ Fire Safety Engineering Group \\ The University of Greenwich \\ Park Row, Greenwich \\ London SE10 9LS, UK
}

\begin{abstract}
Emergency exit signs form an essential part of building wayfinding systems. However, in some emergency evacuation situations many people fail to see the emergency exit signs and in some cases, even if detected by the population, the signs have been ineffective in leading them to safety. These failings are primarily due to their inability to attract the immediate attention of the people they are there to assist and their lack of an ability to respond to a changing threat environment. The need for exit signs that attract attention when they need to be conspicuous, to redirect people to not just an exit route, but a viable and if possible an optimal exit route in an evolving emergency has driven the development of a new generation of advanced signage system, the Intelligent Active Dynamic Signage System (IADSS). The IADSS, developed as part of the European Commission FP7 funded GETAWAY project, attempts to meet these needs through the development of a novel Active Dynamic Signage System (ADSS) which can be controlled by an intelligent decision support system. This paper presents results that refine the effectiveness of the ADSS. When an exit route is considered non-viable, the adapted ADSS provides not only negative information i.e. that an exit is no longer available, but also positive information, i.e. directing the population to an alternative exit. In addition, in situations where a voice alarm system is used in conjunction with the ADSS, it is also important to ensure that the messaging does not potentially contradict the intent of the ADSS. The improved ADSS was tested in full-scale evacuation trials in a rail station. The trials suggest that the improved ADSS with voice alarm messaging that does not contradict the signage system successfully redirected $66 \%$ of the participants to the intended exit.
\end{abstract}

KEYWORDS: human behaviour, egress, human factors, signage, dynamic signage, wayfinding

\section{INTRODUCTION}

Efficient evacuation of passengers from large-scale transport terminals in an emergency can be challenging. The complex layout of these structures makes maintaining spatial awareness and wayfinding difficult. In such circumstances, people often attempt to evacuate via familiar routes or the way they entered the building, bypassing or ignoring alternative means of escape, such as emergency exit routes and emergency exits [1]-[3]. In hazardous situations, such as may result from an accidental fire or a terrorist lead incident, safe evacuation can be even more challenging as the available safe evacuation time may be limited and evacuation routes may be compromised due to the rapidly deteriorating environment.

Throughout the world, emergency signage systems have widely been used in the built environment to assist people in evacuation by conveying directional information relating to escape routes and the location of emergency exits. However, several major tragedies, including include the King's Cross Underground fire (1987), the Düsseldorf Airport fire (1996), the Rhode Island Night Club fire (2003) and the Nairobi Westgate shopping mall terrorist attack (2013) [4]-[7], demonstrate the failure of the legally compliant emergency signage systems to fulfil their intended purpose. In the Rhode Island and Dusseldorf incidents, many people failed to see the emergency exit signs, and either did not utilise appropriate emergency exits, or delayed using them, resulting in tragic consequences in many cases. In the King's Cross, Düsseldorf and Nairobi incidents, the emergency exit signs were incapable of adapting to the developing situation and redirecting people away from exit routes that had become compromised, again resulting in tragic consequences. Even in the WTC 911 (2001) disaster, the evacuation of many people was delayed because they could not find the emergency stairs, despite the exits being marked by emergency signs [8]. The failure of emergency signage systems to fulfil their intended purpose in these tragedies is mainly due to their potential to be overlooked by most people [9] or their inability to redirect people away from danger in 
a dynamic rapidly changing environment, making them inherently unreliable in the situations for which they are intended. These and other tragedies have highlighted the crucial need for improving conventional emergency signage. The need for emergency exit signs that attract attention when they need to be conspicuous, to redirect people in an evolving emergency, and to identify, not just an exit route, but a viable exit route and possibly the optimal exit route, has driven the development of a new generation of advanced signage system.

One issue is how to make emergency exit signs stand out, without significantly changing the signage design, e.g. making them larger, which architects and premises owners would rather avoid. One approach is to add lights to draw people's attention to the sign. Jin et al [10] demonstrated in a series of experiments that flashing the light source within a lit sign made the sign more conspicuous to test subjects than a standard exit sign of the same size and at the same viewing distance. Nilsson et al [11], [12] added flashing green lights above an emergency exit and found that the exit are more likely to be used during evacuation trials. Other trials involving the use of test subjects in virtual reality environments also suggest that the use of flashing lights above final emergency exits increase the detectability of the emergency exit [13], [14] within the virtual test environment and so may also improve detectability in the real world. However, these concepts, while addressing the issue of noticeability do not address the issue of closing off a compromised exit route.

The Intelligent Active Dynamic Signage System (IADSS), developed as part of the European Commission FP7 funded GETAWAY project, attempts to address all the identified signage shortcomings. The project, which ran from November 2011 to October 2014, aimed at developing and demonstrating an innovative emergency signage system capable of the real-time optimal redirection of building occupants during an evolving incident. The IADSS, consisting of an enhanced signage system and an intelligent evacuation route selection support system, were developed through several phases during the project. The first development phase included the introduction of two novel signage design concepts to enhance the standard signage system. These are the Dynamic Signage System (DSS) concept that improves signage detectability through the use of a series of green flashing and running LEDs within the sign (see Figure 1) and the negated signage concept that provides a means of effectively shutting down the exit route through the use of a flashing red cross imposed over the exit sign (see Figure 1). The modified sign appears as a standard emergency exit sign until the alarm is sounded, at which point the DSS is activated, hence the name Active Dynamic Signage System (ADSS). The ADSS allows an exit sign to be activated to either encourage or discourage the use of a particular route or exit [15], [16]. It is noted that the negated signage concept developed for the GETAWAY project was examined by Joakim Olander et al [17] in a later questionnaire based study. Although this research was conducted using fewer participants observing virtual signs, the same conclusion, i.e. the flashing red cross design conveys the most clear dissuasive message, was drawn.
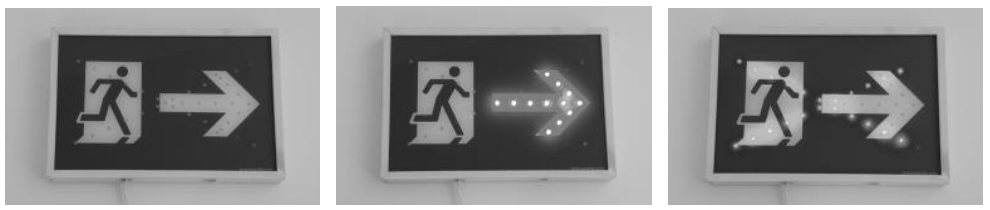

Fig. 1. The standard exit sign (left), the dynamic signage concept (middle) and the negated signage concept (right).

The second development phase brought 'Intelligence' to the automatic control of the ADSS. This is achieved through two distinct support systems developed to work along with the ADSS (see Figure 2). The first is an information gathering system that utilises existing Closed-Circuit Television system (CCTV) and fire detection system to build up-to-the-minute situational awareness. This included the number and distribution of occupants and the presence of fire hazards such as smoke, heat and toxic gases. The population information is collected through the CCTV monitoring the station platform and determined using a people counting algorithm developed by GETAWAY partner Vision Semantics. The state of the fire environment is reported through a simulated fire detection system based on state-of-the-art fire detection system provided by GATEWAY partner Hochiki Europe. The second is an intelligent component known as the Decision Engine (DE) used to identify the optimal exit path. The DE uses the fire signature information provided by the detection system and a pattern matching algorithm developed by FSEG to identify which pre-determined fire from a fire library most closely matches the detected fire signature. The 
fire library contains an extensive range of pre-simulated fires produced using the SMARTFIRE CFD fire simulation software [18]. The population distribution and identified fire are then loaded into the buildingEXODUS evacuation simulation software [9] which proceeds to run through a list of predetermined evacuation strategies for the structure. Using the Safe Egress Route Metric (SERM) algorithm developed by FSEG, the DE interrogates the simulation results and ranks the strategies from best to worst based on factors such as projected fatalities and injuries, distance travelled, evacuation time, number of stairs used, etc. The ranked evacuation strategies are then presented to the human controller who makes the final decision as to which option to implement. The identified exit strategy is then implemented using the ADSS. When activated, the ADSS indicates not only which way to go to reach safety, but just as important, where they should not go. In this way, the IADSS directs occupants to their optimal exits, while at the same time avoiding potential non-viable routes.

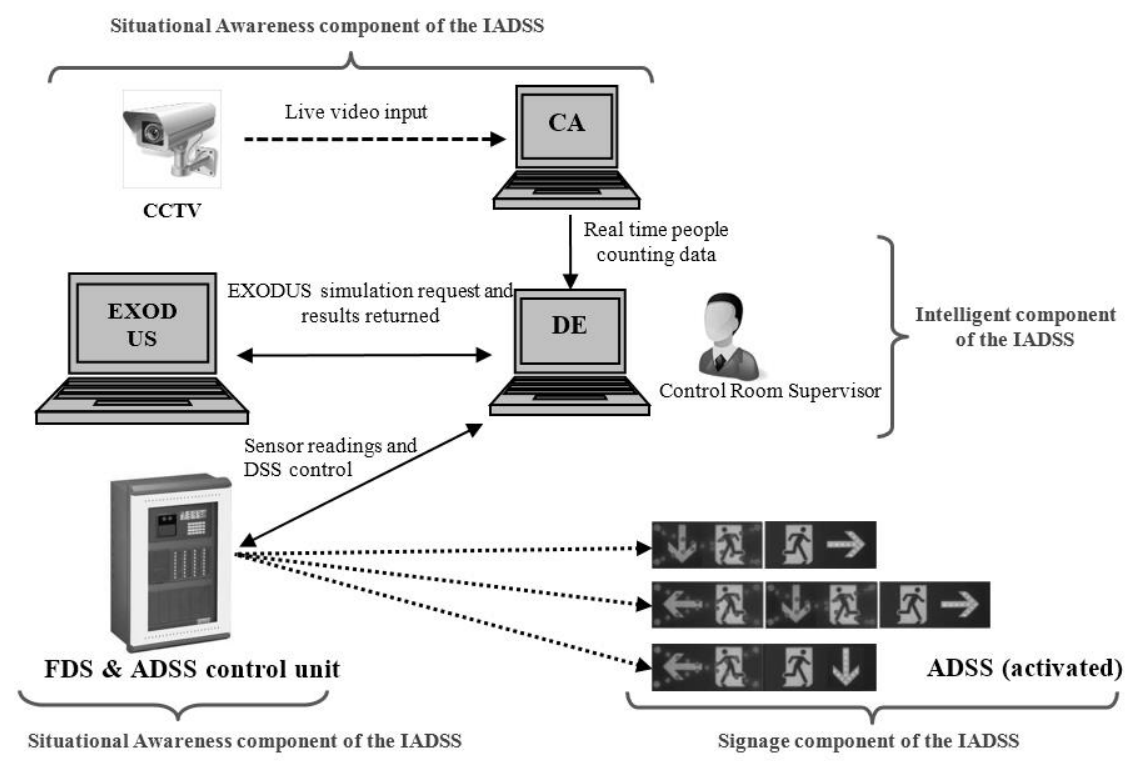

Fig. 2. Setup of the IADSS.

A key component of the IADSS is the ADSS. As part of the GETAWAY project, the ADSS was tested in a rail station to examine its effectiveness in guiding people in a full-scale evacuation trial [15], [16]. In the trial three of the four exits were considered non-viable and so had a negated exit sign located above the exits, while the fourth exit, located at the extreme end of the platform, was considered viable and had the green flashing exit sign located above the exit. Using the ADSS it was demonstrated that it was possible to direct $60 \%$ of the participants to the targeted exit. However, it was noted that some participants, located adjacent to a negated exit and far from the viable exit, did not or could not see the activated sign over the viable exit and so were confused as to which exit to use and so utilized their nearest exit, even though it had a negated exit sign. It was also noted that the voice alarm message directed participants to use their nearest exit which somewhat conflicted with the negative signage information. As a result, three additional largescale trials were planned and conducted to further examine both the effectiveness of the IADSS and a modified signage concept that provided both negative and positive information at each signage location together with a modified voice alarm message. This paper presents the results of these three full scale evacuation trials and a comparison with previous trials to further establish the effectiveness of the modified ADSS concept over the current standard emergency signage systems.

\section{THE EXPERIMENTAL TRIAL METHOD}

As part of the GETAWAY project, three series of trials (TS1, TS2 and TS3) were planned and conducted to assess the effectiveness of the new signage system at three stages of development during the project. In TS1 the ADSS was examined in laboratory settings in which individual participants were instructed to escape a building that has the ADSS installed [19]. The results show that $77 \%$ of the participants 'see' the dynamic sign and $100 \%$ of them go on to follow the sign under relatively ideal conditions. In contrast, in an earlier study the authors demonstrated that standard emergency exit signs were detected by only $38 \%$ of the 
participants [9]. The ADSS was then examined in TS2 at Sant Cugat station in Barcelona, Spain, a functioning train station provided by GATEWAY partner Ferrorcarrils de la Generalitat de Catalunya (FGC) - the operator of the rail system in Barcelona - in order to examine the ADSS within its intended environment (i.e. a realistic application) [15], [16]. In the final phase of the GETAWAY project, the ADSS and the intelligent components were integrated. The entire system was tested through the TS3 trials at the same station. TS3 was partly based on the findings of TS2 and intended to refine and improve the design and application of the ADSS. The results of the TS3 trials were compared with that of the previous trials using the original ADSS (TS2.2) and the conventional escape route signage system (TS2.1) (see Table 1).

Table 1. Full-scale GETAWAY trials.

\begin{tabular}{|c|c|c|c|c|}
\hline $\begin{array}{l}\text { Trial and } \\
\text { conducted } \\
\text { date }\end{array}$ & $\begin{array}{l}\text { Signage } \\
\text { system }\end{array}$ & $\begin{array}{l}\text { Voice } \\
\text { Alarm } \\
\text { message }\end{array}$ & $\begin{array}{l}\text { Number of } \\
\text { participants and } \\
\text { distribution on } \\
\text { platform }\end{array}$ & Signage system and trial description \\
\hline $\begin{array}{c}\text { TS2.1 } \\
(26 / 05 / 13)\end{array}$ & $\begin{array}{l}\text { Conventional } \\
\text { signage } \\
\text { system }\end{array}$ & $\begin{array}{l}\text { Exit rapidly } \\
\text { and use } \\
\text { nearest exit }\end{array}$ & $\begin{array}{l}(139) \\
\text { Boxes 1-7 fully } \\
\text { occupied }\end{array}$ & $\begin{array}{l}\text { Baseline test using conventional emergency exit } \\
\text { signage. Participants distributed along entire } \\
\text { length of platform and signs indicate nearest exit } \\
{[16] \text {. }}\end{array}$ \\
\hline $\begin{array}{c}\text { TS2.2 } \\
(02 / 06 / 13)\end{array}$ & $\begin{array}{l}\text { ADSS with } \\
\text { single } \\
\text { message }\end{array}$ & $\begin{array}{l}\text { Exit rapidly } \\
\text { and use } \\
\text { nearest exit }\end{array}$ & $\begin{array}{l}(152) \\
\text { Boxes 1-7 fully } \\
\text { occupied }\end{array}$ & $\begin{array}{l}\text { ADSS trial, similar trial setup to T2.1, but first } \\
\text { three exits have negated sign while fourth exit, at } \\
\text { extreme end of platform has flashing green exit } \\
\text { sign [16]. }\end{array}$ \\
\hline $\begin{array}{c}\text { TS3.1 } \\
(31 / 05 / 14)\end{array}$ & $\begin{array}{l}\text { Modified } \\
\text { ADSS with } \\
\text { multiple } \\
\text { messages }\end{array}$ & $\begin{array}{l}\text { Exit rapidly } \\
\text { and use } \\
\text { nearest exit }\end{array}$ & $\begin{array}{l}(171) \\
\text { Boxes 1-7 fully } \\
\text { occupied }\end{array}$ & Modified ADSS, similar trial setup to TS2.2. \\
\hline $\begin{array}{c}\text { TS3.2 } \\
(01 / 06 / 14)\end{array}$ & $\begin{array}{l}\text { Modified } \\
\text { ADSS with } \\
\text { multiple } \\
\text { messages }\end{array}$ & Exit rapidly & $\begin{array}{l}\text { (139) } \\
\text { Boxes 1-6 fully } \\
\text { occupied }\end{array}$ & $\begin{array}{l}\text { Modified ADSS, similar trial setup to T3.1 with a } \\
\text { slightly reduced participant population. Number } \\
\text { of participants located adjacent to the intended } \\
\text { exit (Exit D) was reduced. }\end{array}$ \\
\hline $\begin{array}{c}\text { TS3.3 } \\
(01 / 06 / 14)\end{array}$ & $\begin{array}{l}\text { Modified } \\
\text { ADSS with } \\
\text { multiple } \\
\text { messages }\end{array}$ & Exit rapidly & $\begin{array}{l}\text { (64) } \\
\text { Only Box } 1 \text { and } \\
\text { area to its left } \\
\text { were occupied }\end{array}$ & $\begin{array}{l}\text { Modified ADSS, trial involves a reduced } \\
\text { population placed at the opposite end of the } \\
\text { platform to the intended exit (Exit D). }\end{array}$ \\
\hline
\end{tabular}

\section{Trial Design}

The TS3 trials had two design objectives. First, the entire IADSS was tested to examine how its various components worked together to achieve the original design specifications. Most importantly, the performance of the intelligent component of the IADSS was evaluated to determine if, given a hypothetical fire within the station the DE could identify the optimal exit route within the available short time period between the alarm system going from 'alert' mode to 'alarm' mode. Second, the trials were designed to examine the consistency of the performance of the ADSS in guiding people to exit from an unfamiliar environment in a presumed emergency situation, without staff intervention. In this paper we present the results addressing the second objective. As such, the results of TS3 presented here are considered a continuation of TS2 [15], [16]. Before describing TS3 it is worth briefly mentioning the TS2 trials and their main findings [16].

TS2.1 was conducted to establish a baseline response to standard emergency exit signage. The trial involved one platform of the Sant Cugat station which had four exits distributed along the length of the platform. The station staff did not intervene during the evacuation trial but a voice alarm system directing participants to exit via their nearest exit. The trial involved 139 participants who were distributed uniformly along the length of the platform. The results of the trial demonstrated that $99 \%$ of the participants exited the station using their nearest exit. A small number of participants were identified as actively looking at the emergency exit signs. The post-trial questionnaire results confirmed that signage only accounted for $27 \%$ of participant exit selection, while the most significant factor, which accounted for $51 \%$, was the proximity of the exit to the evacuating population. These results suggest that where this signage information was present, although understood, the information provided by the standard emergency exit signage was not as influential as the proximity of an exit in exit selection. 
TS2.2 was conducted to assess the participant reaction to the ADSS. The trial involved the use of the ADSS to direct 152 participants on the same platform towards one of the four exits which was located at one end of the platform. This was not the nearest exit for the majority of the population and therefore required the population to adopt a different behaviour from that exhibited in TS2.1, i.e. not utilise their nearest exit. The results showed that overall, $63 \%$ of the total population used the intended exit; i.e. followed the intended procedure indicated by the ADSS [16]. However, excluding those for which the targeted exit was their nearest exit, only $43 \%$ of participants were redirected to the targeted exit.

As demonstrated through TS2.2, the ADSS was influential in participant exit route choice and was capable of directing a large proportion of the participant population towards a distant target exit and away from closer (non-viable) exits. However, as only positive or negative information was provided at any single signage location, it was noted that while some participants may have perceived the negated exit sign, they had difficulty in perceiving the only positive exit sign located at the extreme end of the platform beside the target exit. As a result, they had no other viable option than to use their nearest exit. Thus while the participants may have correctly interpreted the negated sign, as they could not perceive any other viable exit option, they utilised the negated exit. Furthermore, it was noted that the voice alarm message instructed people to move towards their nearest exit. The exact message was:

*Tone, Tone, Tone*

There is an emergency in the station, please leave the station immediately by your nearest exit *Tone, Tone, Tone*

Repeat message

The alarm voice message could have influenced some of the participants to utilise their nearest exit, reducing the effectiveness of the ADSS. These two issues raised a question in the design and application of the ADSS. Could providing both negative and positive signage information at each sign location and a more appropriate alarm message improve the effectiveness of the ADSS?

Thus, in TS3, the ADSS and the alarm message were modified to address the issues raised by TS2. Firstly, in TS3 the ADSS was redesigned so that it could convey both positive information (identifying an exit that could be used) and negative information (identifying an exit that should not be used) at any single signage location. For this purpose, it is necessary for the sign to point not only to the nearest exit but to the nearest alternative exits. If an exit was considered non-viable, the active dynamic sign would have a corresponding flashing red-cross, but the sign would also indicate the direction to the nearest viable exit(s) through a flashing green arrow. The signs would also need to be coordinated so that they automatically conveyed consistent messages. Figure 3 shows an example of the modified dynamic sign above Exit $\mathrm{C}$ in Figure 4 . In this situation the sign is indicating that the nearest exit, Exit $\mathrm{C}$ is not considered viable, the exit immediately to the left of Exit C (Exit B see Figure 4) is also considered non-viable but the exit to the right of Exit C (Exit D see Figure 4) is viable. The modified sign thus conveys not only that an exit or exits are not viable, but also provides information as to which exit is viable.

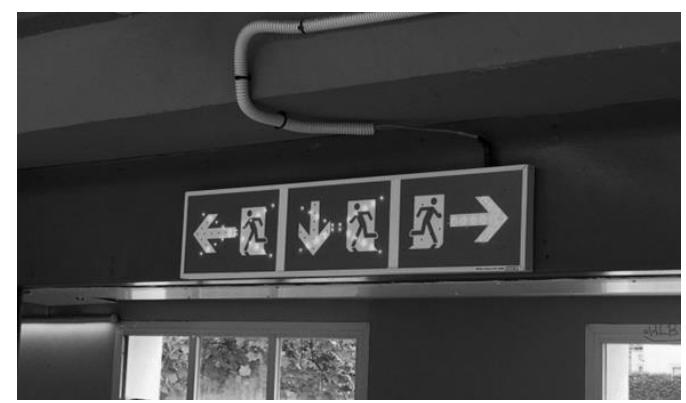

Fig. 3. Dynamic signage configuration example for TS3.

Secondly, the alarm message broadcast through the public address system was modified so that it no longer stated to exit via the nearest exit, but simply to leave immediately. The modified message was changed to:

*Tone, Tone, Tone*

There is an emergency in the station, please leave the station immediately

*Tone, Tone, Tone* 


\section{Repeat message}

Two TS3 trials were originally planned for two successive dates with the intention to run one additional trial on day 2 to test a more challenging scenario. The first TS3 trial TS3.1, was a comparable case to TS2.2 and intended to measure the potential improvement offered by the redesigned ADSS. In order to isolate the potential impact of the alarm message, TS3.1 used the same alarm message used in TS2.2 which instructed people to move towards their nearest exit. The second TS3 trial, TS3.2, used the modified alarm message which instructs people to exit as quickly as possible, but not to suggest using the nearest exit. This trial used a slightly reduced number of participants. Finally, the additional TS3 trial, TS3.3, used the modified alarm message and a more challenging case involving a smaller participant population initially located at the opposite end of the platform to the intended exit. In this trial, the entire group of participants would have to pass by all three negated exits before finally exiting via the intended exit at the far end of the platform. In this way the participants would utilise a complex chain of signs to navigate to the intended exit, passing three negated exits on the way. It should be noted the same modified ADSS was used to implement the same evacuation strategy (i.e. using the only indicated viable exit) across all three TS3 trials.

\section{Trial Location, Scenario, Procedure and Participants}

The trials took place at the Sant Cugat station in Barcelona, Spain. The trials involved one platform of the station (Platform 2) that was occupied by only by the participants. The evacuation scenario (only relevant for the testing of the DE not discussed here) involved a hypothetical luggage fire adjacent to the ticket office. The platform is approximately 38 metres long and has four exits (Exit A to D) from left to right (see Figure 4). Exit A and Exit D lead out of the station onto a public road. Exit B and Exit C are entrances to the underpass which leads to the main entrance on the other side of the station. Exit B is an elevator which would not normally be used as part of an evacuation but was an exit point.

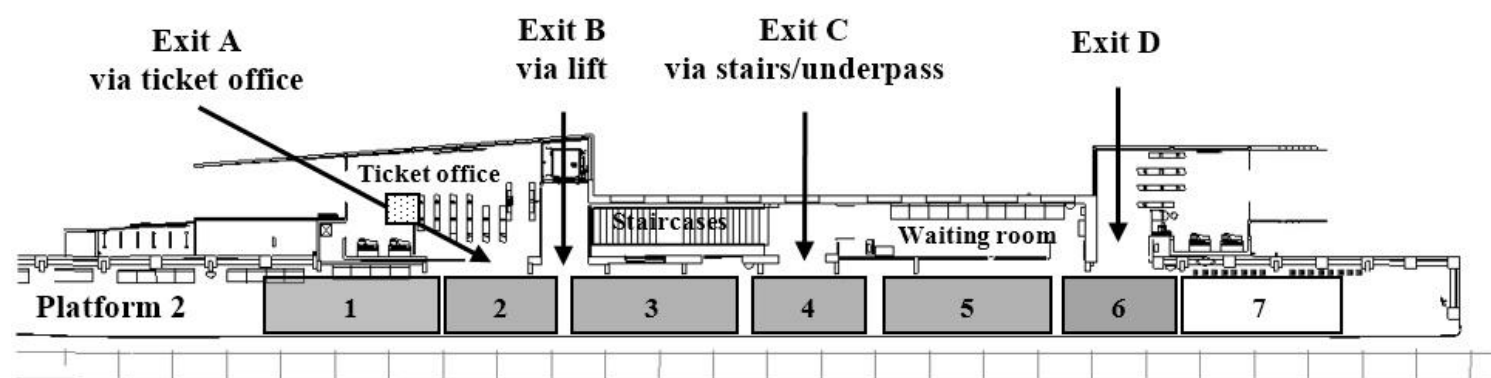

Fig. 4. Layout of Platform 2 of the Sant Cugat station with 7 boxes used to distribute the participants and the location of the assumed luggage fire (dotted square).

To ensure that the conditions in TS3 trials were comparable with TS2 trials, the participants were located in each of seven staging boxes located along the platform (see Figure 4). The population density within the boxes was approximately the same in each box and in each trial and representative of conditions during a busy period $\left(2\right.$ persons $/ \mathrm{m}^{2}$ ). To achieve this, some 200 participants were required for each trial. FGC recruited the participants from the local area using adverts at their stations, on their website, social networks and local media. A total of 1356 volunteers registered their interest in attending the trials. These volunteers were then selected according to the trial requirements, which included limited knowledge of the trial station and the demographics resembling typical FGC travellers, to produce the final participant population.

The number recruited for TS3.1 on day 1 was 229 and the number recruited for TS3.2 and TS3.3 on day 2 was 274 . On each trial day fewer participants arrived than were recruited, with 171 participants attending TS3.1 on day 1 and 203 participants being available for TS3.2 and TS3.3 on day 2. Approximately $26 \%$ of the invited participants failed to show up. Nevertheless, the number of participants were considered sufficient to conduct the TS3 trials. While the demographics of the trials was planned and partially controlled through the selection process, the actual demographics of the populations used in the trials was governed by the number and nature of people that actually showed up on the trial days. The actual demographics of the two populations for day 1 and day 2 are broadly similar and representative of users of 
the FGC system. In addition, the participant populations are also similar in all the other aspects, including the distribution of participants' occupation, participant impairment present during the trials, their level of familiarity with the station and exit awareness. Of the participant populations, about $49 \%$ in all trials were unfamiliar with the station (i.e. they never used the station), while about $37 \%$ were infrequent users of the station (i.e. they used the station less than once per month). To compensate participants for incurred travel costs, they could select one of two forms of compensation consisting of a small cash payment or free rail and entry tickets to an FGC tourist attraction on completing the trials and questionnaires.

The evacuation scenario is driven by a hypothetical fire that occurs in the lobby area of Exit A on Platform 2 (see Figure 4) and makes that exit non-viable. It is also assumed that the fire occurred during rush hour with all passengers evenly distributed across Platform 2 (except trial TS3.3 in which the participants were placed at one end of the platform). As a result of the fire incident, all occupants on the platform need to be safely evacuated via available routes. All exit routes are considered initially viable while the DE of the IADSS assesses the situation and identifies which is the best evacuation route to utilise by the passengers located on the platform, given the location of the fire, the projected spread of the fire hazards and the distribution of passengers. The evacuation strategy identified by the DE was to evacuate all participants on the platform via only Exit D. This strategy was implemented through the automatic activation of the ADSS (see Figure 5). However, the participants were allowed to choose any route they thought appropriate given the information available to them. It is important to note that there was no actual or theatrical fire on the platform and so the participants did not receive additional exiting cues from the fire. Discussion of the DE and the route selection process are beyond the scope of this paper and will be discussed in a later publication.

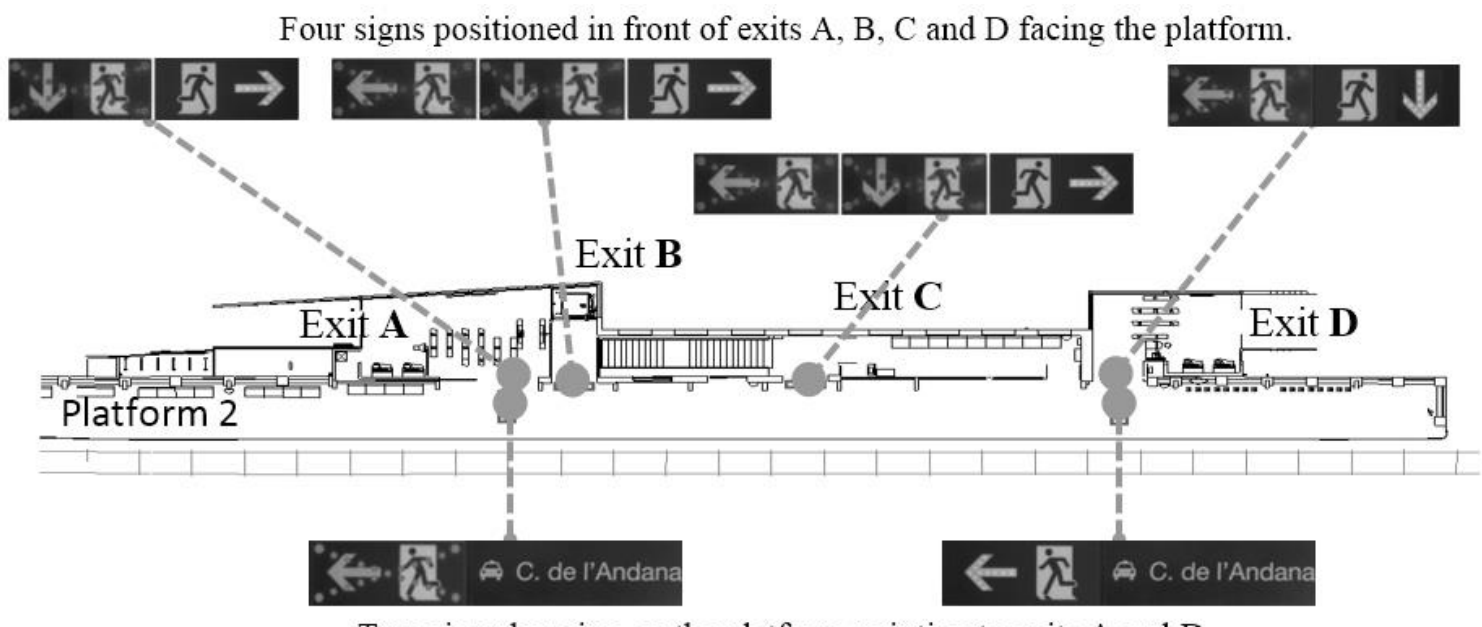

Two signs hanging on the platform pointing to exits $\mathrm{A}$ and $\mathrm{D}$.

Fig. 5. The activated DSS during the TS3 trials according to the selected evacuation strategy.

On the day of each trial, the participants gathered at a nearby station and were briefed about the trial but were given no specific information concerning the signage systems or the purpose of the trials. The participants were taken to the trial station by train and distributed on the platform at specific locations identified by the numbered (and coloured) boxes on the platform (see Figure 4) - thus the participants did not experience using any of the exits prior to the trial. The participants were positioned to face the track as if they were waiting for the next train and waited until the start of the trial which was signified by the sounding of the fire alarm. The fire alarm was a voice alarm with a tone followed by a message instructing the participants that there is an emergency and they should leave the station immediately. This sequence was constantly repeated throughout the trial. Platform 2 was then evacuated. There was no staff intervention to assist or guide the participants during the evacuation.

The routes adopted by the evacuating population during each trial were recorded using video cameras for later analysis. On completion of the trials the participants were given a questionnaire that was designed to collect information regarding participant exit choice, the factors influencing their decision and their opinions concerning the signs they encountered during the trials. The questionnaire was similar to that used in the earlier trials [16] however, the language had been improved to reduce potential ambiguities noted in 
TS2. The data derived from the video footage and the completed questionnaires were cross-referenced where possible in order to increase the credibility of the findings.

It is noted that an independent ethics review of the trial design was carried out by the University of Greenwich independent Research Ethics Committee to ensure that the proposed trials met recognised ethical standards for conducting trials with members of the public and for the collection, analysis and handling of personal data.

\section{EXPERIMENTAL RESULTS AND DISCUSSION}

The results of the three full-scale evacuation TS3 trials are presented and discussed in this section. These trials involved the use of the modified ADSS to direct the participants towards Exit D, according to a specific evacuation strategy, given the nature of the assumed incident.

\section{Results of TS3.1}

Trial TS3.1 involved 171 participants. Presented in Table 2 are the number of participants that started in each of the seven numbered boxes and then used one of the three exits. It should be noted that due to the restricted camera views on the platform, only 168 participants were counted in video footage and involved in the analysis. It should also be noted that TS3.1 used the original alarm message which instructed people to use their nearest exit.

Table 2. Exit usage in TS3.1.

\begin{tabular}{|c|c|c|c|c|c|c|c|c|c|}
\hline \multirow{3}{*}{ Exit used } & \multicolumn{7}{|c|}{ Boxes and initial population size } & \multirow[b]{2}{*}{ Total } & \multirow{3}{*}{$\begin{array}{l}\% \text { moved } \\
\text { to each exit }\end{array}$} \\
\hline & Blue/1 & Magenta/2 & Green/3 & Yellow/4 & Orange/5 & Purple/6 & White/7 & & \\
\hline & 29 & 18 & 26 & 23 & 19 & 22 & 31 & 168 & \\
\hline Exit A & 19 & 14 & 10 & 0 & 0 & 0 & 0 & 43 & $25.6 \%$ \\
\hline Exit B & 0 & 0 & 0 & 0 & 0 & 0 & 0 & 0 & $0 \%$ \\
\hline Exit $\mathrm{C}$ & 0 & 0 & 1 & 12 & 0 & 0 & 0 & 13 & $7.7 \%$ \\
\hline Exit D & 10 & 4 & 15 & 11 & 19 & 22 & 31 & 112 & $66.7 \%$ \\
\hline
\end{tabular}

The results show that the majority of the participants $(66.7 \%, 112$ out of 168$)$ followed the guidance of the ADSS and used Exit D. These 112 participants originated from all seven boxes. Of them, 53 originated from areas that are closer to other exits. Excluding those for whom Exit D was their nearest exit, it is estimated that $48.6 \%$ of the participants followed the ADSS guidance and did not use their nearest exit and.

\section{Results of TS3.2}

In total 203 people turned up for day 2 of the trials, considerably more than the 171 that showed up for day 1 (TS3.1). However, two trials were planned for day 2, TS3.2 and TS3.3. In order to have sufficient numbers of participants for TS3.3 it was decided to reduce the number of participants in TS3.2. Thus, TS3.2 involved 139 participants. This trial was similar in nature to TS3.1 but with the modified alarm message. Given the reduced size of the population in TS3.2 it was decided to maintain the population density in each of the boxes as in TS3.1 but not to populate the $7^{\text {th }}$ box on the platform which is close to Exit D. This was because in the previous trials (TS3.1 and TS2.2), everyone originating from this box utilised Exit D. The participants originally allocated to this box in TS3.2 were allocated, along with the additional participants that showed up for day 2 to TS3.3. Thus in TS3.2, the population density in the boxes used in the trial were similar to those in TS3.2 but the $7^{\text {th }}$ box, at the end of the platform adjacent to Exit D was empty. Presented in Table 3 are the number of participants that started in each of the six numbered boxes and then used one of the three exits.

Again, the results show that the majority of the participants $(66.9 \%, 93$ out of 139) followed the guidance of the ADSS and used Exit D. Of the 93 participants who used Exit D, 63 originated from areas that are closer to other exits. If those for whom Exit D was their nearest exit were excluded, it is estimated that $57.8 \%$ of the participants followed the ADSS guidance and did not use their nearest exit. 
Table 3. Exit usage in TS3.2.

\begin{tabular}{|c|c|c|c|c|c|c|c|c|c|}
\hline \multirow{3}{*}{ Exit used } & \multicolumn{7}{|c|}{ Boxes and initial population size } & \multirow{2}{*}{ Total } & \multirow{3}{*}{$\begin{array}{l}\% \text { moved } \\
\text { to each exit }\end{array}$} \\
\hline & Blue/1 & Magenta/2 & Green/3 & Yellow/4 & Orange/5 & Purple/6 & White/7 & & \\
\hline & 26 & 19 & 20 & 26 & 27 & 21 & - & 139 & \\
\hline Exit $\mathrm{A}$ & 18 & 6 & 2 & 0 & 0 & 0 & - & 26 & $18.7 \%$ \\
\hline Exit B & 0 & 0 & 0 & 0 & 0 & 0 & - & 0 & $0 \%$ \\
\hline Exit $\mathrm{C}$ & 0 & 0 & 0 & 18 & 2 & 0 & - & 20 & $14.4 \%$ \\
\hline Exit D & 8 & 13 & 18 & 8 & 25 & 21 & - & 93 & $66.9 \%$ \\
\hline
\end{tabular}

\section{Results of TS3.3}

Trial TS3.3 involved a smaller population of 64 participants who occupied the Blue/1 box furthest from Exit D and an additional similar sized area immediately adjacent to and on its left at the start of the trial. The alarm message was the same as that in TS3.2 which did not instruct participants to exit via their nearest exit, but to exit as quickly as possible. This trial is considered the most challenging of all the trials conducted as the participants must walk past three apparently viable exits to reach Exit D. Furthermore, the participants do not benefit from observing other participants, at least initially, utilising the only viable exit. Thus the participants must rely entirely on the information provided by the ADSS to find and use the indicated exit. Presented in Table 4 are the number of participants that used one of the three exits.

Table 4. Exit usage in TS3.3.

\begin{tabular}{|c|c|c|c|c|}
\hline \multirow{2}{*}{ Exit used } & \multicolumn{2}{|c|}{ Boxes and initial population size } & \multirow{2}{*}{ Total } & \multirow{2}{*}{$\%$ moved to each exit } \\
\cline { 2 - 4 } & Additional box to the left of Blue/1 box & Blue/1 & & \\
\cline { 2 - 4 } & 32 & 32 & 64 & \\
\hline Exit A & 22 & 22 & $34.4 \%$ \\
\hline Exit B & 0 & 0 & $0 \%$ \\
\hline Exit C & 0 & 0 & $0 \%$ \\
\hline Exit D & 42 & 42 & $65.6 \%$ \\
\hline
\end{tabular}

Similar to the results of TS3.1 and TS3.2, the majority of the participants (65.6\%, 44 out of 64) followed the guidance of the ADSS and used Exit D in TS3.3. Furthermore, of the 42 participants that did not use Exit A, 0\% used Exit B, which was the next nearest exit or Exit $\mathrm{C}$ both of which they had to walk past to reach Exit D. $100 \%$ of these participants continued on to use Exit D. Since all 64 participants originated from areas that are closer to other exits, $65.6 \%$ of the participants did not use their nearest exit and followed the ADSS guidance - the highest proportion among the three TS3 trials.

\section{Questionnaire analysis of participants' interpretation of the ADSS}

The key results from the questionnaires concerning the participants' interpretation of the signage are summarised in this section. The signage interpretation questions required participants to indicate their level of agreement with a set of statements (i.e. the intended meanings of the signs) based on their experiences during the trials using a five point Likert scale, ranging from 'strongly disagree' to 'strongly agree'. The results from TS3.2 and TS3.3 are combined and presented here as these two trials had the same appropriate emergency alarm message. The Agree/Strongly Agree and the Disagree/Strongly Disagree options are also grouped together. Note that the fifth point of the Likert scale not presented in Table 5 represents the neutral option of 'Neither Agree nor Disagree'. Four different ADSS signs were represented in the trials with intended meanings of, 'use this exit', 'don't use this exit' and 'don't use this exit, but use the indicated exit'. Thus the statements being tested were:

"This sign assisted me in selecting (a) an exit to use /(b) which exit NOT to use /(c) which exit not to use and which exit to use."

The results are shown in Table 5. Note that the number of replies are not consistent across all the signs as not all the participants were exposed to all the signs and not all participants recalled seeing the signs. Overall there was a high level of agreement $(81 \%)$ that the signs were effective in indicating which exit to use and which exit not to use. 
Table 5. Participants' level of agreement (TS3.2 and TS3.3) with the intended meanings of the signs.

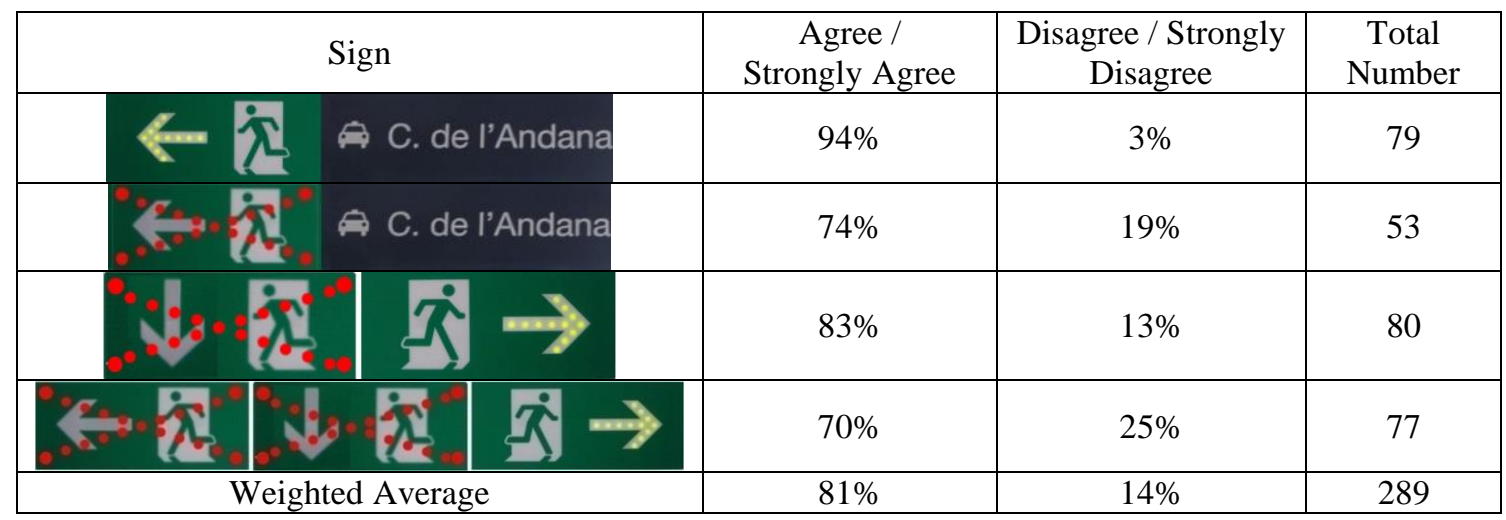

The red cross and arrow were combined for the first time in TS3, therefore this design concept was also tested through the questionnaire to determine whether the participants found the concept confusing. The results from TS3.2 and TS3.3 are combined and presented here as these two trials had the same appropriate emergency alarm message. The Agree/Strongly Agree and the Disagree/Strongly Disagree options are also grouped together. Note that the fifth point of the Likert scale not presented in Table 6 represents the neutral option of 'Neither Agree nor Disagree'. Two different ADSS signs displaying multiple messages were represented in the trials and hence were tested separately (see Table 6). The statement being tested was:

\section{"Overall I felt confused by the combination of flashing red cross and green arrow."}

The results are shown in Table 6. Note that the number of replies are not consistent across all the signs as not all the participants were exposed to all the signs and not all participants recalled seeing the signs.

Table 6. Participants' level of agreement (TS3.2 and TS3.3) that combined cross and arrow confused them.

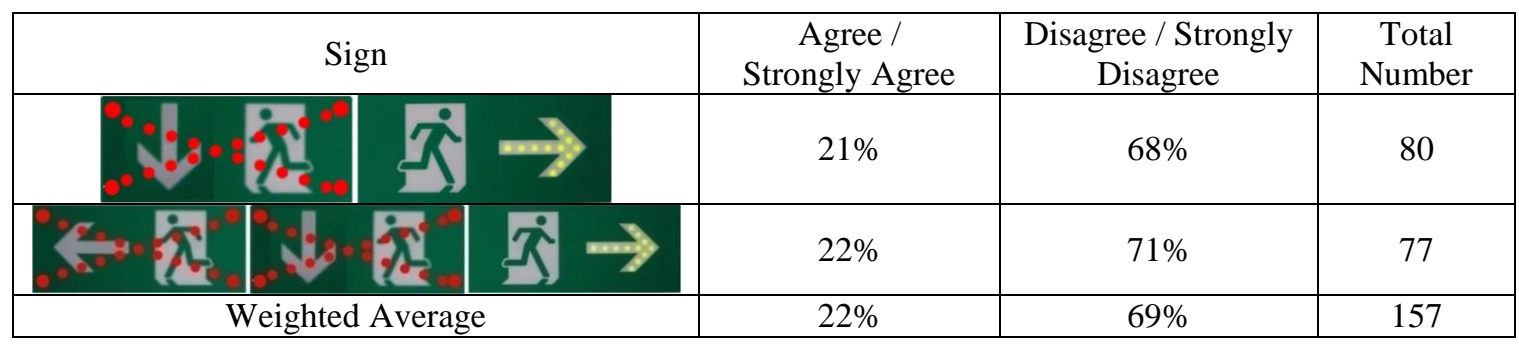

Overall there was a reasonably high level of agreement (69\%) that the signs conveying both positive and negative information were not confusing.

\section{Discussion}

Trial TS2.2 and TS3.1 help to illustrate the difference between the original ADSS design and the modified ADSS design. In both trials, the population was distributed across the entire platform in the same manner and the same alarm message was used. It should be noted that this alarm message instructed participants to use their nearest exit. In TS2.2, only positive or negative information was available at each signage location, while both positive and negative information was available in TS3.1. This change in the ADSS design resulted in a marginal increase of $6 \%$ in the number of participants using the intended exit (see Table 7).

Table 7. Effectiveness of the ADSS in diverting participants to Exit $\mathrm{D}^{*}$.

\begin{tabular}{|c|c|c|c|c|c|}
\hline Trial & TS2.1 & TS2.2 & TS3.1 & TS3.2 & TS3.3 \\
\hline$\%$ moving to nearest Exit & $99 \%$ & $57 \%$ & $51 \%$ & $42 \%$ & $34 \%$ \\
\hline \% moving to Exit D & $43 \%$ & $43 \%$ & $49 \%$ & $58 \%$ & $66 \%$ \\
\hline
\end{tabular}

* Numbers in the table for TS2.2 and TS3 do not include those participants who were initially located near to Exit D. 
The comparison between TS2.2 and TS3.1 demonstrates that while there is a modest improvement in the effectiveness of the ADSS through providing more complete signage information, the conflicting alarm massage diminishes the effectiveness of the ADSS. Nevertheless, it is important to note that $51 \%$ of the population moved to their nearest exit (excluding those who were closest to Exit D) compared with $99 \%$ in the case of the standard signs in TS2.1 (see Table 7).

Trial TS3.1 and TS3.2 further help to illustrate the importance of providing consistent alarm messaging in conjunction with use of the ADSS. In both trials, the population was distributed across the entire platform in the same manner and the same ADSS was used. However, the alarm messaging in TS3.2 instructed the participants to evacuate the station, whereas the alarm messaging in TS3.1 instructed the participants to use their nearest exit. This change in message resulted in an increase of $9 \%$ in the number of participants using the intended exit, even though there were fewer participants placed adjacent to the intended exit (Exit D) in TS3.2. The combined impact of the modified ADSS and non-conflicting voice alarm resulted in a $15 \%$ improvement in the effectiveness of the ADSS. The comparison between TS2.2/TS3.1 and TS3.1/TS3.2 illustrates the importance of providing non-conflicting alarm information when using the ADSS to instruct participants to follow a specific evacuation procedure. Furthermore, it is suggested that the voice alarm message could be used in a positive way to enhance the uptake of the guidance offered by the ADSS by bringing the signage to the attention of the population.

The ADSS had the greatest impact on the participants in TS3.3 where almost two thirds (66\%) of the participants (whose nearest exit was not Exit D) used Exit D. This is in contrast to 58\% in TS3.2, $49 \%$ in TS3.1 and 43\% in TS2.2 (see Table 7). Apart from the difference in the alarm message between TS3.3 and TS2.2/TS3.1, the distribution of the population was different in TS3.3 compared to that in TS2.2/TS3.1. In TS2.2/TS3.1 the participants were evenly distributed across the entire platform, whereas in TS3.3 they were located at the furthest location from the intended exit, Exit D. In TS2.2/TS3.1 a number of participants where initially located directly opposite their nearest exit e.g. the participants in boxes 2,4 and 6. For some of these participants their line of sight may simply not have taken in the signage which is located above their heads over the exit and so they may not have had the opportunity to have been affected by the signage information. As a result, they simply choose to use their nearest exits. This is also the case in TS3.2 which has a similar participant distribution as in TS2.2/TS3.1, although with a more appropriate alarm message and more complete signage information. In TS3.3, all participants started a short distance away from their first nearest exit, Exit A. This potentially allowed them to have more opportunity and time to observe the environment while they were initially approaching Exit A, their nearest exit. Similarly, the participants had to approach Exits $\mathrm{B}$ and $\mathrm{C}$ from a distance providing them with opportunity and time to observe the signs above each of these exits. In this way, the experimental setup in TS3.3 was more like what may be encountered in a building with a signage chain leading occupants to the desired exit point. Even though the targeted exit, Exit D, was located at the furthest end of the platform, requiring the participants to walk past three clearly visible and for all intents and purposes viable exits, the majority of the participants were still willing to follow the ADSS and exit via Exit D. It is thus suggested that the relatively low percentage of the ADSS diverting participants to use a particular exit in TS2.2/TS3.1/TS3.2 should be considered a conservative estimate due to the limitation of positioning participants on the platform as compared with the rate of $66 \%$ in TS3.3.

Concerning the survey analysis, while there was a high level of agreement (81\%) that the ADSS signs were effective in indicating which exit to use and which exit not to use there was some difference in the effectiveness of each sign (see Table 5). The most effective ADSS sign (see Table 5) appears to be the sign conveying the simplest positive information i.e. with the green flashing arrow, with $94 \%$ agreeing or strongly agreeing that the sign assisted them in selecting the exit to use. In comparison, the sign showing the negative information, i.e. which exit not to use, while effective achieving $74 \%$ agreement/strong agreement that it was helpful, was considered somewhat less helpful than the positive signage. This may be because the positive information indicates that a successful exit route has been identified, while the negative information on its own only indicates that an exit is not viable.

Furthermore, when positive and negative information was combined, this also had a high degree of agreement/strong agreement that the information was helpful however, the sign with the least amount of information i.e. a single red cross and a single green arrow was considered helpful by more people (83\%) than the sign with two red crosses and one green arrow (70\%). In addition, the level of agreement is less 
than that for the simple positive information sign. This suggests that signs should be designed to be as simple as possible.

When asked if the ADSS signs conveying both positive and negative information was considered confusing, $69 \%$ of the participants stated that they were not confused. Furthermore, when positive and negative information was combined, there was little difference between the signs based on the amount of information conveyed i.e. one negative and one positive option or two negative and one positive option. However, one fifth still felt that they were confused by the combined signs. This again suggests that a redesign of the green running man emergency sign may be beneficial. Such a design should minimise the amount of information presented when multiple routes are available and simplify the presentation.

\section{Limitations}

As with any experimental project there were several procedural and methodological challenges that arose during the performance of the trials. These limitations should be taken into consideration when reviewing the results.

- Fewer than expected people took part in the trials. The actual population densities achieved during the three TS3 trials were between 1.5 persons $/ \mathrm{m}^{2}$ and 1.7 persons $/ \mathrm{m}^{2}$. This fell short of the desired population densities $\left(2\right.$ persons $/ \mathrm{m}^{2}$ ) which was intended to reflect a busy station platform. This also had the effect of reducing the number of data-points produced for estimating the effectiveness of the signage systems.

- It was difficult to isolate the impact of the signage systems upon route selection from other environmental and social factors, making it impossible to obtain an estimation of the impact of signage systems alone. The attempted solution was to examine the difference in people's escape behaviour under the influence of different signage concepts i.e. standard signs, ADSS signs and modified ADSS signs.

- Height and placement restrictions in positioning the cameras made it difficult to attain ideal monitoring of the population during the trials. This resulted in gaps in the video coverage of the trial area and the difficulty in establishing the interaction between the participants and the signage on the video footage.

- The initial positioning of some of the participants in two of the three trials (TS3.1 and TS3.2) meant that they were located directly opposite or adjacent to their nearest exit. As the emergency signs were located above their heads, this meant that these participants had a significantly reduced opportunity to interact with the emergency signage as it was not in their normal line of sight. This may have adversely impacted the outcome of these trials, suggesting that the reported performance of the ADSS in these trials is more conservative than may normally be expected.

- The questionnaire was formed from a set of 15 high-level closed questions, leading to more probing (although still closed) sub-questions in several instances. By their nature, closed questionnaires only allow the researcher to ask the participant to select between pre-determined responses. Furthermore, while the participants were required to complete the questionnaire immediately after the trials, the answers may be subject to incorrect recollection of their experiences.

- The volunteers were selected so that they had little or no prior knowledge of the station layout, removing familiarity with the layout as a factor influencing exiting behaviour. Thus the vast majority of participants in the trial were unfamiliar with the layout of the station, with about $49 \%$ never having been to the station before and another $37 \%$ having been to the station less than once or twice per month. Thus the trial results only apply to people who are relatively unfamiliar with the layout. It is not clear whether or not the dynamic signage would have been as effective with a population of habitual users of the station.

\section{CONCLUSIONS}

As part of the EU FP7 GETAWAY project, a novel and innovative signage system, known as the Active Dynamic Signage System (ADSS) has been developed that is intended to direct building occupants away from non-viable exit routes and lead them to a safe exit. The ADSS concept involves incorporating two 
novel signage designs into the standard green running man emergency exit sign: a means to attract the attention of building occupants and a means to identify that a previously viable exit route is no longer considered viable. To attract the attention of the building occupants involves incorporating a green flashing and running arrow into the exit sign. Previous work had suggested the ADSS could direct $63 \%$ of the population to exit via the targeted exit, but this included members of the population whose nearest exit was the targeted exit and so would be expected to utilise the exit regardless. However, if only the proportion of the population whose nearest exit was not the targeted exit is considered, then the ADSS was successful in redirecting $43 \%$ of the population towards the targeted exit.

It was suggested that the ADSS could be made more effective in directing people away from non-viable exits if in addition to providing negative information, positive information indicating the location of an alternative exit was also provided. Modifying the ADSS to provide both negative and positive information improved the redirection rate from $43 \%$ to $49 \%$. However, when the voice alarm was also modified so that it no longer suggested that occupants exit via their nearest exit - which may have contradicted the information provided by the ADSS - the redirection rate further improved to 58\%. Finally, in situations where trial participants had ample opportunity to view the emergency signage i.e. they were not located directly beneath or adjacent to the emergency sign, and a non-contradictory alarm message was used, the successful redirection rate was further improved to $66 \%$.

These results clearly demonstrate that the effectiveness of the ADSS in directing a population to a targeted exit can be greatly enhanced if when providing negative information, indicating that an exit route is no longer viable, positive information, indicating an alternative viable exit route, is also provided. Furthermore, the best results are achieved when the voice alarm does not potentially contradict signage messaging but reinforces the signage information. It is thus essential that the nature of the voice alarm messaging is reviewed when used in conjunction with an ADSS. Indeed, utilizing a voice message that instructs the population to follow the guidance of the signage system may further improve the effectiveness of the ADSS.

Results from the participant survey demonstrated that overall there was a high level of agreement (81\%) that the ADSS signs were effective in indicating which exit to use and which exit not to use. However, the highest level of agreement was achieved for signs that conveyed the simplest information - single green flashing arrow (94\%) - while the least level of agreement was achieved for signs that conveyed the most complex information - two flashing red crosses and one flashing green arrow (70\%). Nevertheless, one fifth of the sample (22\%) suggested that they found the signs conveying the multiple information confusing. This suggests that a redesign of the green running man emergency sign may be beneficial where it is necessary to convey multiple route information. It is suggested that alternative designs should attempt to minimize the amount of information presented when multiple routes are available and simplify the presentation.

\section{ACKNOWLEDGEMENT}

Project GETAWAY (contract 265717) was funded under the European Union Framework 7 Transport initiative. The authors acknowledge the co-operation of their project partners: BMT, Vision Semantics Ltd, London Underground Ltd, Evaclite Ltd, Hochiki Europe, Kingfell Bulgaria and FGC Spain; in undertaking this work and in allowing the project findings to be published. The authors are particularly grateful to FGC for allowing the use of two of their railway stations on two days.

\section{REFERENCES}

[1] Sime, J.D., 1985. Movement Towards the Familiar - Person and Place Affiliation in a Fire Entrapment Setting, Environment and Behaviour, 17(6): 697-724, doi:10.1177/0013916585176003.

[2] Shields T.J. and Boyce K.E., 2000. A Study of Evacuation from Large Retail Stores, Fire Safety Journal 35, 2000, 25-49. doi:10.1016/S0379-7112(00)00013-8.

[3] Nilsson, D., Frantzich, H., Saunders, W., 2008. Influencing Exit Choice in the Event of a Fire Evacuation., Fire Safety Science - Proceedings of the ninth Symposium, IAFSS, 2008, pp 341352. http://dx.doi.org/10.3801/IAFSS.FSS.9-341. 
[4] Fennell, D., 1998. Investigation into the King's Cross Underground Fire, Her Majesty's Stationery Office: London, ISBN 0-10-104992-7

[5] Weinspach, P.M., Gundlach, J., Klingelhofer, H.G., Ries, R., Schneider, U., 1997. Analysis Of The Fire On April 11th, 1996. Recommendations And Consequences For Düsseldorf Rhein-RuhrAirport, Staatskanzlei Nordrhein-Wstfalen, Mannesmannufer 1 A, 40190 Düsseldorf, Germany

[6] Grosshandler, W., Bryner, N., Madrzykowski, D. and Kuntz K., 2005. Report of the Technical Investigation of the Station Nightclub Fire, NIST NCSTAR 2: Vol. I-II, National Institute of Standard and technology, Gaitherburg, MD., USA

[7] BBC, 2013. Nairobi siege: How the attack happened. [Online] Available at: http://www.bbc.co.uk/news/world-africa-24189116

[8] Galea, E.R., 2014. High-Rise Building Evacuation Post 911 - Addressing the Issues. Tall Building Fire Safety Conference 2014, Proceedings of the 2nd International Conference, CMS Press, Greenwich, UK, ISBN 978190452853, pp 75-89, 2014

[9] Xie, H., Filippidis, L., Galea, E.R., Blackshields, D., Lawrence, P.J., 2012. Experimental analysis of the effectiveness of emergency signage and its implementation in evacuation simulation, Fire and Materials, Vol 36, Issue 5-6, pp. 367-382, DOI: 10.1002/fam.1095

[10] Jin, T., Yamada, T., Kawai, S. and Takahashi, S., 1991. Evaluation Of The Conspicuousness Of Emergency Exit Signs. Fire Safety Science 3: 835-841. doi:10.3801/IAFSS.FSS.3-835

[11] Nilsson, D., Frantzich, H., \& Saunders, W., 2005. Coloured Flashing Lights to Mark Emergency Exits - Experiences from Evacuation Experiments. Fire Safety Science - Proceedings of the Eighth International Symposium, International Association of Fire Safety Science, pp 569-579.

[12] Nilsson, D., Frantzich, H., \& Saunders, W., 2008. Influencing Exit Choice in the Event of a Fire Evacuation. Fire Safety Science - Proceedings of the Ninth International Symposium, International Association of Fire Safety Science, pp 341-352. DOI:10.3801/IAFSS.FSS.9-341.

[13] Enrico Ronchi, Daniel Nilsson, 2015. A Virtual Reality experiment on the design of flashing lights at emergency exit portals for road tunnel evacuations. Lund University Report, Report 3180, 2015.

[14] Emília Duarte, Francisco Rebelo, Júlia Teles, Michael S. Wogalter, 2014. Behavioral compliance for dynamic versus static signs in an immersive virtual environment., Applied Ergonomics, Vol 45, pp 1367-1375, http://dx.doi.org/10.1016/j.apergo.2013.10.004

[15] Galea, E.R., Xie, H., Cooney, D. and Filippidis, L., 2015, Active Dynamic Signage System: A Full-Scale Evacuation Trial, Human Behaviour in Fire, Proceedings 6th Int Symp 2015, Interscience Communications Ltd, London, ISBN 978-0-9933933-0-3, pp 303-314, Sept 2015.

[16] Galea, E.R., Xie, H., Deere, S., Cooney, D. and Filippidis, L., 2016, An International Survey and Full-Scale Evacuation Trial Demonstrating the Effectiveness of the Active Dynamic Signage System Concept, Fire and Materials, (accepted for publication).

[17] Joakim Olander, Enrico Ronchi, Ruggiero Lovreglio, Daniel Nilsson, 2017, Dissuasive exit signage for building fire evacuation, Applied Ergonomics, Volume 59, Part A, March 2017, Pages 84-93

[18] Xiaoqin Hu, Zhaozhi Wang, Fuchen Jia, Edwin R Galea, Mayur K Patel, 2011, Simulating Smoke Transport in Large Scale Enclosure Fires using a Multi-Particle-Size Model, Proceedings of the Tenth International Symposium on Fire Safety Science, University of Maryland, 19-24 June 2011, pp. 445-458. ISSN 1817-4299. DOI: 10.3801/IAFFS.FSS.10-445

[19] Galea, E.R., Xie, H. and Lawrence, P.J., 2014. Experimental and Survey Studies on the Effectiveness of Dynamic Signage Systems, Fire Safety Science 11: 1129-1143. 10.3801/IAFSS.FSS.11-1129 